\title{
Linear Dispersion Codes for Limited Feedback Channels with Feedback Impairments
}

\author{
Fernando M. L. Tavares, Renato Machado, Bartolomeu F. Uchôa-Filho, and André N. Barreto
}

\begin{abstract}
Linear dispersion codes (LDCs) are an attractive tool for attaining spatial diversity in OFDM systems with multiple transmit antennas. To reach the potential gains, in each situation, the selection of a proper set of codes depends, in general, on the existence of a reliable feedback channel. We propose LDCs for multiple-input multiple-output (MIMO) channels with a fixed amount of feedback. The proposed scheme selects the LDC from a set of LDCs that minimizes the error probability based on the instantaneous channel conditions. The determination of the best set of LDCs is described as a constrained optimization problem. We present several good sets of LDCs, obtained from an optimization algorithm. Through simulation results we show that the proposed schemes outperform previously reported comparable results, yet at the price of increased complexity. We also show the performance gains that can be reached in practice with LDC, by critically evaluating the feedback channel impairments, such as errors and signalling delays, and also considering the effect of signalling overhead on the achievable throughput.
\end{abstract}

Index Terms-Limited feedback channel, linear dispersion codes, MIMO, OFDM, transmit diversity.

\section{INTRODUCTION}

M ULTIPLE-INPUT MULTIPLE-OUTPUT (MIMO) wireless communication systems can exploit the spatial dimension to achieve higher transmission rates and/or to improve the error performance over fading channels. For single-input multiple-output (SIMO) wireless channels, techniques such as selection diversity combining (SDC), equal gain combining (EGC), and maximum ratio combining (MRC) have been used to obtain diversity gain. One way of obtaining the benefits of the spatial dimension for wireless channels with multiple transmit antennas is through spacetime coding [1], which requires no knowledge of the channel state information (CSI), i.e., the fading coefficients associated with the channels from the transmit to the receive antennas, at the transmitter. For MISO or MIMO wireless channels, the performance can be considerably improved if a feedback channel exists so that the CSI is known at the transmitter. If the CSI is perfectly known at the transmitter, then maximum ratio transmission (MRT) [2] is the optimum beamforming. If the antenna amplifiers are required not to modify the

F. M. L. Tavares is with the Nokia Institute of Technology (INdT), Brasília, DF, 70397-900, BRAZIL. E-mail: ftees.aau.dk. R. Machado is with the Signal Processing and Communications Research Group, Department of Electronics and Computing, Federal University of Santa Maria, Santa Maria, RS, 97105-900, BRAZIL. E-mail: renatomachado@ufsm.br. B. F. Uchôa-Filho is with the Communications Research Group, Department of Electrical Engineering, Federal University of Santa Catarina, Florianópolis, SC, 88040-900, BRAZIL. E-mail: uchoa deel . ufs c. br. André N. Barreto is with the Department of Electrical Engineering, University of Brasília, Brasília, DF, 88040-900, BRAZIL. E-mail: andrebarreto@ene.unb. br

This paper was presented in part at the IEEE Wireless Communications and Networking Conference (WCNC'08), Las Vegas, USA, March-April 2008. transmit powers of the transmitted signals, then equal gain transmission (EGT) [3] with any combining scheme (SDC, EGC or MRC) achieves full diversity order over the MIMO flat fading channel.

A more realistic assumption, however, is that of limited feedback (consisting of a few bits) with partial CSI at the transmitter. This situation requires some form of quantization of either the CSI or the beamforming vectors. The most usual approach is to define a finite codebook of possible beamforming vectors known to both the transmitter and receiver, and based on the instantaneous CSI the receiver only needs to send through the feedback channel the label of the best (according to a given criterion) beamforming vector. The analysis of quantization methods for MIMO channels with limited feedback is a well-studied research problem [4]-[8].

In [6], the codebook design problem was shown to be equivalent to the problem of packing one-dimensional subspaces known as Grassmannian line packing. Although Grassmannian beamforming achieves full diversity for the case of i.i.d. Rayleigh fading channels, no practical algorithm to construct the codebook was developed. One important contribution of [6] was the derivation of a bound on the number of feedback bits required to achieve full diversity. In particular, for $M_{T}$ transmit antennas, at least $\log _{2}\left(M_{T}\right)$ bits are required.

The problem of quantizing the EGT (Q-EGT) beamforming vector was first proposed in [5], and recently addressed by Murthy and Rao [7]. In [7], a vector quantization approach to Q-EGT is considered, and an algorithm based on the generalized Lloyd algorithm is presented that converges to a possibly local optimum codebook in the sense of maximizing capacity. The theoretical results developed in [7] are valid when the SNR is either very high or very low, and progressively becomes more accurate as the number of feedback bits gets larger.

In [8], assuming that the transmitter uses the quantized information for beamforming, the authors derive a universal lower bound on the outage probability for any finite set of beamformers. By this result, it is possible to characterize the gain with each additional bit of feedback information regarding the channel state, and to show how the performance approaches that of perfect CSI.

Besides the theoretical contributions to this important subject, as referenced earlier, a number of practical schemes designed for the MIMO wireless channels with limited feedback have been proposed, many of them adopting the minimization of the instantaneous probability of error, or, equivalently, maximization of the signal-to-noise ratio (SNR) [9]-[16].

An interesting feedback-assisted scheme, proposed by Gore and Paulraj [9], uses an orthogonal space-time block code 
(OSTBC) [17] with the best subset of antennas, selected from $M_{T} \geq 3$ transmit antennas available. They have shown that a transmit diversity order of $M_{T}$ is achieved, as if all the transmit antennas were used. Herein we refer to this scheme as transmit antenna selection/Alamouti (TAS/A) when the Alamouti code is used.

Chen et al. [10] have presented an error probability analysis for the so called transmit antenna selection/maximal-ratio combining (TAS/MRC). In this scheme, which we refer to as TAS/T ("T" stands for trivial), a single transmit antenna is selected from $M_{T} \geq 2$ transmit antennas. Again, a transmit diversity order of $M_{T}$ is achieved.

Similar ideas have been proposed by Machado and UchôaFilho [11], refined later in [12], [13]. They have proposed a hybrid transmit antenna/code selection scheme that chooses from a list of (orthogonal and non-orthogonal) STBCs the best code to be used with a subset of transmit antennas. In the same direction, the so called group-coherent codes (GCCs) have been proposed by Akhtar and Gesbert [14], [15] for $M_{T}=p M$ transmit antennas and $p-1$ feedback bits. A GCC for $M_{T}=p M$ transmit antennas consists of a STBC for the first $M$ transmit antennas and the same STBC for each one of the other $p-1$ groups of $M$ transmit antennas, where the signals transmitted through each group (except the first one) of $M$ antennas is multiplied by either +1 or -1 , the polarities being determined by $p-1$ feedback bits. The transmit diversity order achieved is $p M$ and, again, the aim is to minimize the instantaneous error probability. Each multiplying factor \pm 1 can be seen as a 2-level quantization of $\exp (j \theta)$. Each of the $p-1$ feedback bits determines a phase $\theta \in\{0, \pi\}$. In other words, the multiplying factors are taken from BPSK. In [16], the GCCs have been extended to yield a better error performance with $(p-1) \log _{2}(r)$ feedback bits, where $r$ is some positive power of 2 .

In these practical schemes, the set of codes have not been optimized formally, and their choice was based on intuition. In this paper, for wireless channels with $M_{T} \geq 2$ transmit antennas, $M_{R}$ receive antennas, and limited feedback, we propose a code selection (CS) transmission scheme that uses the best code from a set of $2^{b_{f}}$ codes, where $b_{f}$ is the number of feedback bits. By best, we mean the code that minimizes the instantaneous error probability.

The codes in the set of possible selections belong to the broad class of linear dispersion codes (LDCs), introduced in [18]. LDCs are good candidates for high-rate MIMO signaling over wireless channels. Transmitted codewords of an LDC are formed by linear combinations over space and time of certain dispersion matrices, the transmitted data symbols being the combining coefficients. Our preference for the LDCs is due to the fact that encoding and decoding are an easy task when these codes are used, and constitute a broad class of codes, of which many space-time codes, antenna/code selection schemes, etc. are particular cases.

Herein, for a fixed amount of feedback, the determination of the best set of LDCs, i.e., the one that minimizes the average error probability, is described as a constrained optimization problem. A closed-form solution to this problem is difficult to obtain. Nevertheless, for certain values of $M_{T}, b_{f}$, and codeword length, we obtained good sets of LDCs, by using an optimization algorithm. Although our results are concentrated on sets of rate-one LDCs, sets with codes of other rates can be sought as well. Also, results are shown for $M_{R}=1$ receive antenna, but the results are valid and can easily be adapted to the MIMO case with any combining scheme (SDC, EGC or MRC). This is the first contribution of this paper. Part of this contribution was presented in [19].

We call attention to the fact that the LDCs in the good sets given in this paper correspond to a form of uniform QEGT, although some sets may have a more general form as compared to the ones in [7], because herein the number of per-antenna bits may be different for different antennas. Since for some parameters the optimization algorithm outputs the uniform Q-EGT solution, the results in this paper support the claim made in [7, Section VII] that the uniform quantization is in fact very likely to be the optimum scalar quantized when the total number of feedback bits is a multiple of $\left(M_{T}-1\right)$.

However, those LDCs were not studied in more realistic conditions where limitations of the feedback channel are present. Firstly, even though the feedback channel conveys quantized information, it is still not possible to send the index of the code word for each symbol or, in an OFDM system, for each carrier. Therefore, the feedback information is usually just related to a sample of the whole channel. Secondly, errors and delays are limitations of any communication channel and the feedback channel is not an exception. In this paper, the LDC presented in [19] is analyzed taking into account these inherent feedback channel limitations and it will be shown that gains of the LDC technique can only be obtained when some conditions are met. We investigate the performance not only in terms of the bit error rate, but also consider the goodput, where we include the signalling overhead from the feedback channel. This is the second contribution of this paper.

The paper is organized as follows: In Section II, we review LDCs. The proposed scheme and its associated optimization problem are described in Section III. In Section IV, we describe the optimization algorithm used to obtain the good sets of LDCs for selected parameters given in Section V. In Section VI, we present simulation results of several LDC codes considered in Section V. In Section VII, the results of the analysis of the feedback channel limitations are presented and discussed. Finally, Section VIII concludes the paper.

\section{LINEAR DISPERSION CODES}

In this section, we present the model for MIMO systems employing LDCs. Consider a MIMO system with $M_{T}$ transmit and $M_{R}$ receive antennas. Assume that the channel has a flat Rayleigh fading and remains constant over $\tau$ symbol intervals. The input-output relationship is given by

$$
\mathbf{Y}=\gamma_{0} \mathbf{X H}+\mathbf{W},
$$

where $\mathbf{Y}$ is the $\tau \times M_{R}$ matrix of the received signals and $\mathbf{X}$ is the $\tau \times M_{T}$ matrix of transmitted signals with unit average energy. Let $\mathcal{C N}(0, \mathbf{R})$ represent the joint p.d.f. (probability density function) of a zero-mean circularly symmetric complex normal random vector with covariance matrix $\mathbf{R}$. Then, $\mathbf{W}$ is 
the $\tau \times M_{R}$ matrix $\mathcal{C N}\left(\mathbf{0}, \mathbf{I}_{\tau M_{R}}\right)$, representing the joint p.d.f. of the i.i.d. additive Gaussian noise samples with unit variance, $\mathbf{H}$ is the $M_{T} \times M_{R}$ MIMO channel characterized by the p.d.f. $\mathcal{C N}\left(\mathbf{0}, \mathbf{I}_{M_{T} M_{R}}\right)$, and $\gamma_{0}=\sqrt{\frac{\rho}{M_{T}}}$, with $\rho$ the average SNR at each receive antenna, regardless of the number of transmit antennas.

Assume we have $Q r$-QAM data symbols $\left\{s_{q}\right\}, q=$ $1, \ldots, Q$, with unit average energy, to be transmitted over $\tau$ symbol intervals. The linear transmission (LD) matrix $\mathbf{X}$ is then given by [18]

$$
\mathbf{X}=\sum_{q=1}^{Q} \alpha_{q} \mathbf{A}_{q}+j \beta_{q} \mathbf{B}_{q},
$$

where we have decomposed the transmitted symbol $s_{q}$ into its real and imaginary parts, i.e., $s_{q}=\alpha_{q}+j \beta_{q} .\left\{\mathbf{A}_{q}, \mathbf{B}_{q}\right\}$, $q=1, \ldots, Q$, are complex-valued dispersion matrices of dimension $\tau \times M_{T}$ that specify the code. The rate of the LDC is

$$
R=\frac{Q \log _{2} r}{\tau}(\text { bit/symbol interval) } .
$$

Typically, the dispersion matrices $\left\{\mathbf{A}_{q}, \mathbf{B}_{q}\right\}, q=1, \ldots, Q$, are required to satisfy the following energy constraints, listed in increasing order of stringency [18]:

$$
\begin{gathered}
\sum_{q=1}^{Q} \operatorname{Tr}\left(\mathbf{A}_{q}^{\mathcal{H}} \mathbf{A}_{q}+\mathbf{B}_{q}^{\mathcal{H}} \mathbf{B}_{q}\right)=2 \tau M_{T} ; \\
\operatorname{Tr} \mathbf{A}_{q}^{\mathcal{H}} \mathbf{A}_{q}=\operatorname{Tr} \mathbf{B}_{q}^{\mathcal{H}} \mathbf{B}_{q}=\frac{\tau M_{T}}{Q}, \quad q=1, \ldots, Q ; \\
\mathbf{A}_{q}^{\mathcal{H}} \mathbf{A}_{q}=\mathbf{B}_{q}^{\mathcal{H}} \mathbf{B}_{q}=\frac{\tau}{Q} I_{M_{T}}, \quad q=1, \ldots, Q
\end{gathered}
$$

where $(\cdot)^{\mathcal{H}}$ represents conjugate transpose and $\operatorname{Tr}(\mathbf{A})$ denotes the trace of matrix $A$. In (4) we normalize the total transmit power, (5) and (6) guarantee that the transmitted power is distributed equally among all $Q$ symbols and all transmit antennas, respectively.

Now, let us define $\mathbf{Y}_{R} \triangleq \Re\{\mathbf{Y}\}$ and $\mathbf{Y}_{I} \triangleq \Im\{\mathbf{Y}\}$. Denote the $n$-th column of $\mathbf{Y}_{R}, \mathbf{Y}_{I}, \mathbf{H}_{R}, \mathbf{H}_{I}, \mathbf{W}_{R}$ and $\mathbf{W}_{I}$, respectively, by $\mathbf{y}_{R, n}, \mathbf{y}_{I, n}, \mathbf{h}_{R, n}, \mathbf{h}_{I, n}, \mathbf{w}_{R, n}$ and $\mathbf{w}_{I, n}$, and define

$$
\begin{gathered}
\mathcal{A}_{q} \triangleq\left[\begin{array}{cc}
\mathbf{A}_{R, q} & -\mathbf{A}_{I, q} \\
\mathbf{A}_{I, q} & \mathbf{A}_{R, q}
\end{array}\right], \quad \mathcal{B}_{q} \triangleq\left[\begin{array}{cc}
-\mathbf{B}_{I, q} & -\mathbf{B}_{R, q} \\
\mathbf{B}_{R, q} & -\mathbf{B}_{I, q}
\end{array}\right], \\
\underline{\mathbf{h}}_{n} \triangleq\left[\begin{array}{c}
\mathbf{h}_{R, n} \\
\mathbf{h}_{I, n}
\end{array}\right]
\end{gathered}
$$

where $n=1, \ldots, M_{R}$, and $\mathbf{A}_{R, q}, \mathbf{A}_{I, q}, \mathbf{B}_{R, q}$, and $\mathbf{B}_{I, q}$ are the real and the imaginary parts of the matrices $\mathbf{A}_{q}$ and $\mathbf{B}_{q}$, respectively. With these definitions, we rewrite (1) as:

$$
\underbrace{\left[\begin{array}{c}
\mathbf{y}_{R, 1} \\
\mathbf{y}_{I, 1} \\
\vdots \\
\mathbf{y}_{R, M_{R}} \\
\mathbf{y}_{I, M_{R}}
\end{array}\right]}_{\mathbf{y}}=\sqrt{\frac{\rho}{M_{T}}} \mathcal{H} \underbrace{\left[\begin{array}{c}
\alpha_{1} \\
\beta_{1} \\
\vdots \\
\alpha_{Q} \\
\beta_{Q}
\end{array}\right]}_{\mathbf{x}}+\underbrace{\left[\begin{array}{c}
\mathbf{w}_{R, 1} \\
\mathbf{w}_{I, 1} \\
\vdots \\
\mathbf{w}_{R, M_{R}} \\
\mathbf{w}_{I, M_{R}}
\end{array}\right]}_{\mathbf{w}},
$$

where the equivalent $2 M_{R} \tau \times 2 Q$ real-valued channel matrix $\mathcal{H}$ is given by

$$
\mathcal{H}=\left[\begin{array}{ccccc}
\mathcal{A}_{1} \underline{\mathbf{h}}_{1} & \mathcal{B}_{1} \underline{\mathbf{h}}_{1} & \ldots & \mathcal{A}_{Q} \underline{\mathbf{h}}_{1} & \mathcal{B}_{Q} \underline{\mathbf{h}}_{1} \\
\vdots & \vdots & \ddots & \vdots & \vdots \\
\mathcal{A}_{1} \underline{\mathbf{h}}_{M_{R}} & \mathcal{B}_{1} \underline{\mathbf{h}}_{M_{R}} & \ldots & \mathcal{A}_{Q} \underline{\mathbf{h}}_{M_{R}} & \mathcal{B}_{Q} \underline{\mathbf{h}}_{M_{R}}
\end{array}\right] .
$$

The LDCs subsume, as special cases, both V-BLAST [20] and orthogonal STBC [17]. From (2), we can see that LDCs are very simple to encode. Furthermore, LDCs can be decoded very efficiently by several well-known MIMO demodulation algorithms, such as the sphere decoder [21], the sequential Monte Carlo (SMC)-based detector [22], the nulling-andcanceling detector [23], as well as the simple linear detectors [24].

\section{LiNEAR Dispersion CODES FOR MIMO ChanNELS WITH LIMITED FEEDBACK}

In this section, we design LDCs for MIMO channels with limited feedback. Consider the MIMO system in Section II with $M_{T} \geq 2$ transmit antennas and, for simplicity, $M_{R}=1$ receive antenna. Assume the channel fading coefficients are known at the receiver, and that an error-free and a delayfree feedback channel is available through which $b_{f}$ bits can be sent to the transmitter. In our transmission scheme, based on the instantaneous channel conditions, the receiver determines which LDC within a set of $2^{b_{f}}$ LDCs yields the maximum instantaneous SNR (i.e., minimum instantaneous error probability). It then sends a $b_{f}$-bit index through the feedback channel indicating the best LDC to the transmitter.

Let us define the complete real and imaginary dispersion matrices $\mathbf{A}=\left[\mathbf{A}_{1}, \mathbf{A}_{2} \cdots \mathbf{A}_{Q}\right]$ and $\mathbf{B}=\left[\mathbf{B}_{1}, \mathbf{B}_{2} \cdots \mathbf{B}_{Q}\right]$, both of dimension $\tau \times\left(M_{T} Q\right)$. Also, let $J=\left\{1, \ldots, 2^{b_{f}}\right\}$ be an index set, $\mathcal{S}=\{\{\mathbf{A}(i), \mathbf{B}(i)\}: i \in J\}$ be a set containing $2^{b_{f}}$ LDCs and $\mathcal{H}(\{\mathbf{A}(i), \mathbf{B}(i)\}, \mathbf{H})$ be the matrix in (9) for the $i$-th LDC in $\mathcal{S}$ for a given channel realization $\mathbf{H}$. The instantaneous pairwise error probability (PEP), i.e., the probability conditioned on $\mathcal{H}$ that a maximum-likelihood (ML) receiver decides erroneously in favor of a real vector e given that the real data vector $\mathbf{x}$ defined in (8) was transmitted is

$$
P(\mathbf{x} \rightarrow \mathbf{e} \mid \mathcal{H})=P\left(\left\|\mathbf{y}-\gamma_{0} \mathcal{H} \mathbf{e}\right\|^{2}<\left\|\mathbf{y}-\gamma_{0} \mathcal{H} \mathbf{x}\right\|^{2}\right),
$$

which is well approximated by [1]

$$
P(\mathbf{x} \rightarrow \mathbf{e} \mid \mathcal{H}) \leqslant \exp \left(-\|\mathcal{H}(\mathbf{x}-\mathbf{e})\|^{2} \frac{\gamma_{0}^{2} M_{T}}{4}\right),
$$

where $\|\cdot\|^{2}$ denotes the Frobenius norm. The probability in (10) depends not only on the signal constellation adopted but also on the specific pair of correct and erroneous vectors, which makes the solution to our problem more difficult to obtain. Instead, we shall be interested in a representative error performance measure that depends essentially on the instantaneous channel realization $\mathbf{H}$ and on the description of the LDC offered by the dispersion matrices $\mathbf{A}$ and $\mathbf{B}$, regardless of the constellation adopted. That is, we consider the PEP conditioned on $\mathcal{H}$ derived in [18]

$$
P_{e}(\mathcal{H}) \leq \frac{1}{2} \operatorname{det}\left[I+\frac{\gamma_{0}^{2}}{2} \mathcal{H}^{T} \mathcal{H}\right]^{-1 / 2},
$$


where $(\cdot)^{T}$ denotes transpose. This formula is based on the Gaussian input assumption, so it is not dependent on a particular signal constellation. For a channel realization $\mathbf{H}$, and for a given set $\mathcal{S}$ of LDCs, in our transmission scheme the receiver selects the index $i^{*}$ corresponding to the best LDC according to

$$
i^{*}=\arg \min _{i} P_{e}(\mathcal{H}(\{\mathbf{A}(i), \mathbf{B}(i)\}, \mathbf{H})) .
$$

Let us now describe the optimization problem related to the determination of the optimal set of LDCs. We want to find the set of LDCs that minimizes the average PEP over all channel realizations. Let

$$
\bar{P}_{e}(\mathcal{S})=E_{\mathbf{H}} \min _{\{\mathbf{A}, \mathbf{B}\} \in \mathcal{S}} P_{e}(\mathcal{H}(\{\mathbf{A}, \mathbf{B}\}, \mathbf{H}))
$$

be the average PEP for the set $\mathcal{S}$ of LDCs. We should note that an $\operatorname{LDC}\{\mathbf{A}, \mathbf{B}\}$ is a point in the $\mathbb{C}^{2 \tau M_{T} Q}$ space. So a set $\mathcal{S}$ is a point in $\underbrace{\mathbb{C}^{2 \tau M_{T} Q} \times \cdots \times \mathbb{C}^{2 \tau M_{T} Q}}_{2^{b_{f}} \text { times }}$. Then the optimal set $\mathcal{S}^{*}$ of LDCs (subject to one of the constraints in Sec. II) is given by

$$
\mathcal{S}^{*}=\arg \underbrace{\mathbb{C}^{2 \tau M_{T} Q} \underbrace{\min }_{\times \cdots \times \mathbb{C}^{2 \tau M_{T} Q}}}_{2^{b_{f}} \text { times }} \bar{P}_{e}(\mathcal{S}) .
$$

As observed in [25], the minimization of the expected value of (12) cannot be solved analytically. The optimization problem in (15), which is far more complex, must thus be solved by some computational method. In particular, the continuous multidimensional complex space in which the dispersion matrices reside should be restricted to some finite subset, and expectations with regard to $\mathbf{H}$ should be obtained through simulations over a large number of independent channel realizations.

\section{Optimization Algorithm}

Finding the optimum set of LDCs for each system configuration (parameters $M_{T}, M_{R}, \tau, Q$ and $b_{f}$ ) is a complex task. An exhaustive search can be done over all possible set combinations, but it becomes unfeasible as the number of combinations increases, and, as far as we are aware, there is no analytical solution to this problem.

In this section we present the search algorithm that was considered to find the sets of LDCs presented in Section V.

An optimization algorithm was proposed in [16], where the power restrictions in (5) and (6) should always be satisfied. In this section, we propose an algorithm that may consider only the restriction in (4), decreasing the algorithm complexity, and making it possible to use when restrictions (5) and (6) are not required. We also reduce the algorithm complexity by eliminating, at an early stage, all the LDCs that provide a high PEP, and are thus not likely to be part of the optimum LDC codebook.

The goal of the algorithm is to find the optimum set of $2^{b_{f}}$ dispersion matrix pairs $\{\mathbf{A}(i), \mathbf{B}(i)\}$ that satisfies (15).

In this paper, we represent the dispersion matrix pair by a single complex dispersion matrix $\mathbf{C}(i)=\mathbf{A}(i)+j \mathbf{B}(i)$, in which the element at the $m$-th row and $n$-th column is given by

$$
c_{m, n}(i)=\gamma_{m, n}(i) e^{\theta_{m, n}(i)} .
$$

Thus, the elements of the dispersion matrices $\mathbf{A}$ and $\mathbf{B}$ are

$$
\begin{aligned}
& a_{m, n}(i)=\gamma_{m, n}(i) \cos \theta_{m, n}(i) \\
& b_{m, n}(i)=\gamma_{m, n}(i) \sin \theta_{m, n}(i)
\end{aligned}
$$

To limit the search space we must choose initially a set $\mathcal{S}_{\theta}$ of possible phases $\theta_{m, n}(i)$ and a set $\mathcal{S}_{\gamma}$ of all possible amplitudes $\gamma_{m, n}(i)$. In this contribution we have selected the sets $\mathcal{S}_{\theta}=\{0, \pi / 2, \pi, 3 \pi / 2\}$ and $\mathcal{S}_{\gamma}=\{0, \sqrt{1 / 2}, 1, \sqrt{3 / 2}, \sqrt{2}\}$. However, this choice was arbitrary and not optimized in any particular way. A study of the ideal set size and element values still needs to be performed. The algorithm is described in Algorithm 1 using pseudo-code.

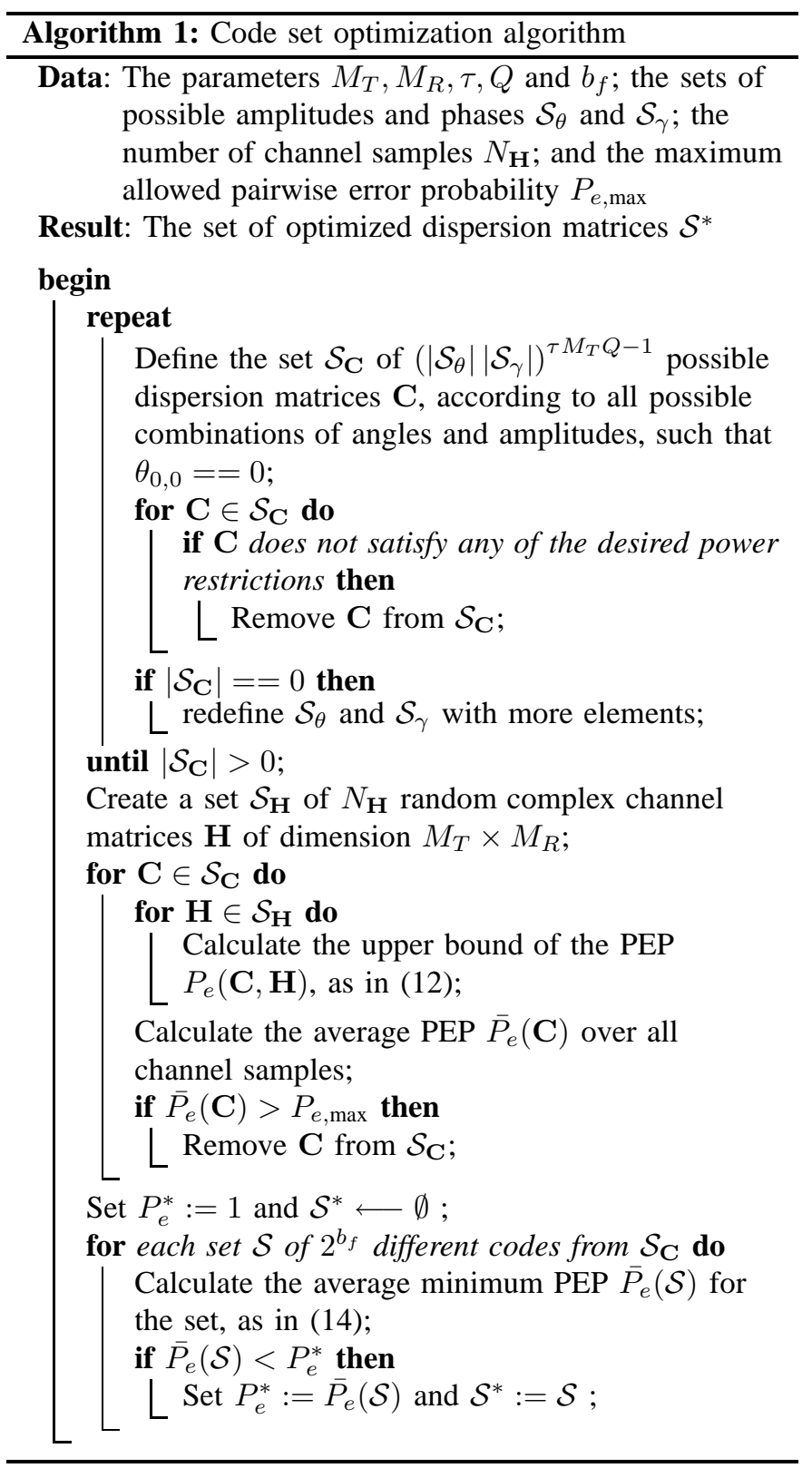


The number of channel samples $N_{\mathbf{H}}$ and the maximum allowed PEP $P_{e, \max }$ for any dispersion matrix are two parameters that can be selected to reduce the algorithm processing time. $N_{\mathbf{H}}$ must be large enough to guarantee statistical confidence and in this work we have considered $N_{\mathbf{H}}=20.000$ channel realizations. However, it is important to mention that we have not performed any investigation on whether a smaller number of channel samples would also provide meaningful results.

Alternatively, instead of setting a maximum $P_{e, \max }$, we can equivalently select the $N_{\mathbf{C}}$ codes with the lowest average PEP. This was the approach taken in this contribution, in which for $M_{T}=2, M_{R}=1, \tau=Q=1$ and $b_{f}=2$, we selected the best 36 dispersion matrices before determining the optimum set.

Even though we cannot guarantee that the codes found with this algorithm are mathematically optimal, we shall see in Section VI that they perform as good as or even better than codes previously found in the literature.

\section{Several Good Sets of LDCs with Feedback}

Herein, the results obtained through the algorithm described in the Section IV are presented, where the power restriction in (5) was considered. At the end of this section, some extensions are also mentioned.

The resulting dispersion matrices for $M_{T}=2, M_{R}=1$, $\tau=Q=1$, and $b_{f}=1$ are $^{1}$

$$
\mathbf{A}_{1}(1)=\mathbf{B}_{1}(1)=\left[\begin{array}{l}
1 \\
1
\end{array}\right]^{T}, \quad \mathbf{A}_{1}(2)=\mathbf{B}_{1}(2)=\left[\begin{array}{c}
1 \\
-1
\end{array}\right]^{T} .
$$

The corresponding codes are

$$
\mathbf{X}_{1}=\left[\begin{array}{ll}
s_{1} & s_{1}
\end{array}\right], \quad \mathbf{X}_{2}=\left[\begin{array}{ll}
s_{1} & -s_{1}
\end{array}\right] .
$$

For $M_{T}=2, M_{R}=1, \tau=Q=1, b_{f}=2$ we have used the same algorithm mentioned earlier (adapted for this new configuration) to find the "best" set of codes. The dispersion matrices of the best set of LDCs (under the energy constraint in (5)) were found to be

$$
\begin{aligned}
& \mathbf{A}_{1}(1)=\mathbf{B}_{1}(1)=\left[\begin{array}{l}
1 \\
1
\end{array}\right]^{T}, \quad \mathbf{A}_{1}(2)=\mathbf{B}_{1}(2)=\left[\begin{array}{l}
1 \\
j
\end{array}\right]^{T}, \\
& \mathbf{A}_{1}(3)=\mathbf{B}_{1}(3)=\left[\begin{array}{c}
1 \\
-1
\end{array}\right]^{T}, \mathbf{A}_{1}(4)=\mathbf{B}_{1}(4)=\left[\begin{array}{c}
1 \\
-j
\end{array}\right]^{T},
\end{aligned}
$$

and the corresponding codes are

$$
\begin{array}{ll}
\mathbf{X}_{1}=\left[\begin{array}{ll}
s_{1} & s_{1}
\end{array}\right], & \mathbf{X}_{2}=\left[\begin{array}{ll}
s_{1} & j s_{1}
\end{array}\right], \\
\mathbf{X}_{3}=\left[\begin{array}{ll}
s_{1} & -s_{1}
\end{array}\right], & \mathbf{X}_{4}=\left[\begin{array}{ll}
s_{1} & -j s_{1}
\end{array}\right] .
\end{array}
$$

We note that the best solution for these simple examples are particular cases of the so-called generalized group-coherent codes [16]. For other parameters, the best solutions are still unknown. However, it should be noted that, with the set of

\footnotetext{
${ }^{1}$ It is important to note that equivalent solutions are possible. Any pair of LD matrices that have the form

$$
\mathbf{A}_{1}(1)=\mathbf{B}_{1}(1)=\left[\begin{array}{l}
a \\
a
\end{array}\right]^{T}, \quad \mathbf{A}_{1}(2)=\mathbf{B}_{1}(2)=\left[\begin{array}{c}
b \\
-b
\end{array}\right]^{T},
$$

is equivalently a good pair of LDCs, where $a$ and $b$ can be arbitrary complex numbers under the energy constraints presented in Section II.
}

LDCs described in (18), the transmitter sends signal $s_{1}$ through antenna 1 and either $s_{1}$ or a version of $s_{1}$ rotated by $\pi$ radians through antenna 2 , depending on the phase difference between $h_{1,1}$ and $h_{2,1}$. In other words, the feedback bit carries phase-only information. This idea can be extended for other parameters, when the number of LDCs in $\mathcal{S}$ (given by $2^{b_{f}}$ ) is matched (in a sense that will become clear) to the number of transmit antennas. Although not obtained by solving the optimization problem in (15), the following sets of rate-one (i.e., $\tau=Q$ ) LDCs are likely to be optimal.

For $M_{T}=2, \tau=Q=1$, and $b_{f}=b$, the associated dispersion matrices are given by

$$
\mathbf{A}_{k, 1}=\mathbf{B}_{k, 1}=\left[\begin{array}{c}
1 \\
\exp \left(j \frac{2 \pi}{2^{b}}(k-1)\right)
\end{array}\right]^{T},
$$

and the corresponding codes are

$$
\mathbf{X}_{k}=\left[\begin{array}{ll}
s_{1} & \exp \left(j \frac{2 \pi}{2^{b}}(k-1)\right) s_{1}
\end{array}\right],
$$

where $k=1, \ldots, 2^{b}$.

Extending for $M_{T}=2, b_{f}=b$, and $\tau=Q \geq 1$, we have that the linear dispersion matrices, for $q=1, \ldots, Q$, are given by

$$
\mathbf{A}_{k, q}=\mathbf{B}_{k, q}=\left[\begin{array}{ccccc}
0 & \ldots & 1 & \ldots & 0 \\
0 & \ldots & \exp \left(j \frac{2 \pi}{2^{b}}(k-1)\right) & \ldots & 0
\end{array}\right]^{T},
$$

where the only nonzero row is the $q$-th row, and the corresponding codes are

$$
\mathbf{X}_{k}=\left[\begin{array}{cc}
s_{1} & \exp \left(j \frac{2 \pi}{2^{b}}(k-1)\right) s_{1} \\
\vdots & \vdots \\
s_{Q} & \exp \left(j \frac{2 \pi}{2^{b}}(k-1)\right) s_{Q}
\end{array}\right]
$$

where $k=1, \ldots, 2^{b}$.

For $M_{T}=3, \tau=Q \geq 1$, and $b_{f}=2$, the linear dispersion matrices, for $q=1, \ldots, Q$, are given by

$$
\begin{aligned}
\mathbf{A}_{1, q} & =\mathbf{B}_{1, q}=\left[\begin{array}{lllll}
0 & \ldots & 1 & \ldots & 0 \\
0 & \ldots & 1 & \ldots & 0 \\
0 & \ldots & 1 & \ldots & 0
\end{array}\right]^{T}, \\
\mathbf{A}_{2, q}=\mathbf{B}_{2, q} & =\left[\begin{array}{lllll}
0 & \ldots & 1 & \ldots & 0 \\
0 & \ldots & 1 & \ldots & 0 \\
0 & \ldots & -1 & \ldots & 0
\end{array}\right]^{T}, \\
\mathbf{A}_{3, q} & =\mathbf{B}_{3, q}=\left[\begin{array}{ccccc}
0 & \ldots & 1 & \ldots & 0 \\
0 & \ldots & -1 & \ldots & 0 \\
0 & \ldots & 1 & \ldots & 0
\end{array}\right]^{T}, \\
\mathbf{A}_{4, q} & =\mathbf{B}_{4, q}=\left[\begin{array}{llcll}
0 & \ldots & 1 & \ldots & 0 \\
0 & \ldots & -1 & \ldots & 0 \\
0 & \ldots & -1 & \ldots & 0
\end{array}\right]^{T},
\end{aligned}
$$

where the only nonzero row is the $q$-th row, and the corresponding codes will be of the form

$$
\begin{array}{ccc}
\mathbf{X}_{1}=\left[\begin{array}{ccc}
s_{1} & s_{1} & s_{1} \\
\vdots & \vdots & \vdots \\
s_{Q} & s_{Q} & s_{Q}
\end{array}\right], & \mathbf{X}_{2}=\left[\begin{array}{ccc}
s_{1} & s_{1} & -s_{1} \\
\vdots & \vdots & \vdots \\
s_{Q} & s_{Q} & -s_{Q}
\end{array}\right], \\
\mathbf{X}_{3}=\left[\begin{array}{ccc}
s_{1} & -s_{1} & s_{1} \\
\vdots & \vdots & \vdots \\
s_{Q} & -s_{Q} & s_{Q}
\end{array}\right], & \mathbf{X}_{4}=\left[\begin{array}{ccc}
s_{1} & -s_{1} & -s_{1} \\
\vdots & \vdots & \vdots \\
s_{Q} & -s_{Q} & -s_{Q}
\end{array}\right] .
\end{array}
$$


For $M_{T}=3, b_{f}=2 b$, and $\tau=Q \geq 1$, we have that the linear dispersion matrices, for $q=1, \ldots, Q$, are of the form $\mathbf{A}_{k, q}$ and $\mathbf{B}_{k, q}$, for $q=1, \ldots, Q$, will be of the form

$$
\mathbf{A}_{k, q}=\mathbf{B}_{k, q}=\left[\begin{array}{ccccc}
0 & \ldots & 1 & \ldots & 0 \\
0 & \ldots & \alpha_{1} & \ldots & 0 \\
0 & \ldots & \alpha_{2} & \ldots & 0
\end{array}\right]^{T},
$$

where the only nonzero row is the $q$-th row, and the corresponding codes will be of the form

$$
\mathbf{X}_{k}=\left[\begin{array}{ccc}
s_{1} & \alpha_{1} s_{1} & \alpha_{2} s_{1} \\
\vdots & \vdots & \vdots \\
s_{Q} & \alpha_{1} s_{Q} & \alpha_{2} s_{Q}
\end{array}\right]
$$

where for each $\mathbf{X}_{k}, k=1, \ldots, 2^{2 b}$, a different pair $\left(\alpha_{1}, \alpha_{2}\right)$ is taken from:

$$
\left\{\exp \left(j \frac{2 \pi}{2^{b}} n_{1}\right), \exp \left(j \frac{2 \pi}{2^{b}} n_{2}\right)\right\}
$$

where $n_{1}, n_{2}=0,1, \ldots, 2^{b}-1$.

As a final and more general extension, we consider any $M_{T} \geq 2, b_{f}=\left(M_{T}-1\right) b$, and $Q \geq 1$. We have that the LD matrices, for $q=1, \ldots, Q$, are of the form

$$
\mathbf{A}_{k, q}=\mathbf{B}_{k, q}=\left[\begin{array}{ccccc}
0 & \ldots & 1 & \ldots & 0 \\
0 & \ldots & \alpha_{1} & \ldots & 0 \\
\vdots & & \vdots & & \vdots \\
0 & \ldots & \alpha_{M_{T}-1} & \ldots & 0
\end{array}\right]^{T},
$$

where the only nonzero row is the $q$-th row, and the corresponding codes will be of the form

$$
\mathbf{X}_{k}=\left[\begin{array}{cccc}
s_{1} & \alpha_{1} s_{1} & \ldots & \alpha_{M_{T}-1} s_{1} \\
\vdots & \vdots & & \vdots \\
s_{Q} & \alpha_{1} s_{Q} & \ldots & \alpha_{M_{T}-1} s_{Q}
\end{array}\right]
$$

where for each $\mathbf{X}_{k}, k=1, \ldots, 2^{\left(M_{T}-1\right) b}$, a different $\left(\alpha_{1}, \ldots, \alpha_{M_{T}-1}\right)$ is taken from:

$$
\left\{\exp \left(j \frac{2 \pi}{2^{b}} n_{1}\right), \ldots, \exp \left(j \frac{2 \pi}{2^{b}} n_{M_{T}-1}\right)\right\}
$$

where $n_{1}, \ldots, n_{M_{T}-1}=0,1, \ldots, 2^{b}-1$.

The codes obtained in this section are referred throughout the rest of the paper as CS/LDC-T codes. A very important observation we can make about them is that, although we have considered in our optimization problem the large class of LDCs, the best codes turned out to be a very special cases of LDCs and are surprisingly simple. The performance of some LDCs is presented in the next section.

\section{Results: Ideal FeEdBack Channel}

In this section, we compare the LDCs discussed in Section $\mathrm{V}$ in terms of their bit error rate (BER) versus $\operatorname{SNR}\left(\gamma_{0}\right)$ for BPSK modulation over a flat quasi-static Rayleigh fading and ideal feedback channel. Regarding the sets of LDCs proposed in this paper, there are certain characteristics that should be mentioned. For instance, only one RF chain is required since the same symbol, up to an antenna-dependent phase rotation, is transmitted through all antennas. Moreover, a very simple linear decoding method with a small decoding delay ( $\tau=1$ for the new LDCs simulated herein) is adopted. The results are presented in Figure 1 for $M_{T}=2$ transmit antennas and in Figure 2 for $M_{T}=3$ transmit antennas, both with $\tau=Q=1$ symbol and $M_{R}=1$ receive antenna. In both figures, the corresponding optimal beamforming scheme is also included.

TABLE I

SET OF CODES OF THE GBLP-2 SCHEME. $M_{T}=2$ AND $b_{f}=2$.

\begin{tabular}{ccc}
\hline & Antenna 1 & Antenna 2 \\
\hline Code 1 & $s_{1}(-0.1612-j 0.7348)$ & $s_{1}(-0.5135-j 0.4128)$ \\
\hline Code 2 & $s_{1}(-0.0787-j 0.3192)$ & $s_{1}(-0.2506+j 0.9106)$ \\
\hline Code 3 & $s_{1}(-0.2399+j 0.5985)$ & $s_{1}(-0.7641-j 0.0212)$ \\
\hline Code 4 & $s_{1}(-0.9541)$ & $s_{1}(0.2996)$ \\
\hline
\end{tabular}

TABLE II

Set of CODES OF THE GBLP-3 SCHEME. $M_{T}=2$ AND $b_{f}=3$.

\begin{tabular}{ccc}
\hline & Antenna 1 & Antenna 2 \\
\hline Code 1 & $s_{1}(0.8393-j 0.2939)$ & $s_{1}(-0.1677+j 0.4256)$ \\
\hline Code 2 & $s_{1}(-0.3427+j 0.9161)$ & $s_{1}(0.0498+j 0.2019)$ \\
\hline Code 3 & $s_{1}(-0.2065+j 0.3371)$ & $s_{1}(0.9166+j 0.0600)$ \\
\hline Code 4 & $s_{1}(0.3478-j 0.3351)$ & $s_{1}(0.2584+j 0.8366)$ \\
\hline Code 5 & $s_{1}(0.1049+j 0.6820)$ & $s_{1}(0.6537+j 0.3106)$ \\
\hline Code 6 & $s_{1}(0.0347-j 0.2716)$ & $s_{1}(0.0935-j 0.9572)$ \\
\hline Code 7 & $s_{1}(-0.7457+j 0.1181)$ & $s_{1}(-0.4553-j 0.4719)$ \\
\hline Code 8 & $s_{1}(-0.7983+j 0.3232)$ & $s_{1}(0.500+j 0.0906)$ \\
\hline
\end{tabular}

TABLE III

SET OF CODES OF THE GBLP-3 SCHEME. $M_{T}=2$ AND $b_{f}=3$.

\begin{tabular}{ccc}
\hline & Antenna 1 & Antenna 2 \\
\hline Code 1 & $s_{1}(0.8393-j 0.2939)$ & $s_{1}(-0.1677+j 0.4256)$ \\
\hline Code 2 & $s_{1}(-0.3427+j 0.9161)$ & $s_{1}(0.0498+j 0.2019)$ \\
\hline Code 3 & $s_{1}(-0.2065+j 0.3371)$ & $s_{1}(0.9166+j 0.0600)$ \\
\hline Code 4 & $s_{1}(0.3478-j 0.3351)$ & $s_{1}(0.2584+j 0.8366)$ \\
\hline Code 5 & $s_{1}(0.1049+j 0.6820)$ & $s_{1}(0.6537+j 0.3106)$ \\
\hline Code 6 & $s_{1}(0.0347-j 0.2716)$ & $s_{1}(0.0935-j 0.9572)$ \\
\hline Code 7 & $s_{1}(-0.7457+j 0.1181)$ & $s_{1}(-0.4553-j 0.4719)$ \\
\hline Code 8 & $s_{1}(-0.7983+j 0.3232)$ & $s_{1}(0.500+j 0.0906)$ \\
\hline
\end{tabular}

TABLE IV

Set of COdes of THE GBLP-4 SCHEME. $M_{T}=3$ AND $b_{f}=2$.

\begin{tabular}{cccc}
\hline & Antenna 1 & Antenna 2 & Antenna 3 \\
\hline Code 1 & $s_{1} / \sqrt{3}$ & $s_{1} / \sqrt{3}$ & $s_{1} / \sqrt{3}$ \\
\hline Code 2 & $j s_{1} / \sqrt{3}$ & $-s_{1} / \sqrt{3}$ & $-j s_{1} / \sqrt{3}$ \\
\hline Code 3 & $-s_{1} / \sqrt{3}$ & $s_{1} / \sqrt{ } 3$ & $-s_{1} / \sqrt{ } 3$ \\
\hline Code 4 & $-j s_{1} / \sqrt{3}$ & $-s_{1} / \sqrt{3}$ & $j s_{1} / \sqrt{3}$ \\
\hline
\end{tabular}

An important class of codes for MIMO channel with limited feedback, named Grassmannian beamforming (Love and Heath [6]), is considered in this section for performance comparisons. Tables I-V present the schemes GBLP-2, GBLP3, GBLP-4, and GBLP-5 [6], respectively, where GBLP means Grassmannian beamforming line packing. In Table V, $\theta_{1}=$ $2 \pi / 3$ and $\theta_{2}=4 \pi / 3$.

Figure 1 considers the TAS/T [10], for $b_{f}=1$ feedback bit, the CS/LDC-T-B and CS/LDC-T-Q, for $b_{f}=1,2$ feedback bits, respectively, and the GBLP-2 and GBLP-3 [6], for $b_{f}=2,3$ feedback bits, respectively. The CS/LDC-T-B and CS/LDC-T-Q schemes are described in (18) and (19), respectively, and can be represented generally by $\left[\begin{array}{ll}s & \alpha_{1} s\end{array}\right]$, 
TABLE V

Set of COdes of THE GBLP-5 SCHEME. $M_{T}=3, b_{f}=3$.

\begin{tabular}{cccc}
\hline & Antenna 1 & Antenna 2 & Antenna 3 \\
\hline Code 1 & $s_{1} / \sqrt{2}$ & $s_{1} / \sqrt{2}$ & 0 \\
\hline Code 2 & $s_{1} / \sqrt{2}$ & 0 & $s_{1} / \sqrt{2}$ \\
\hline Code 3 & 0 & $s_{1} / \sqrt{2}$ & $s_{1} / \sqrt{2}$ \\
\hline Code 4 & $\frac{s_{1} \exp \left\{j \theta_{1}\right\}}{\sqrt{2}}$ & $\frac{s_{1} \exp \left\{j \theta_{2}\right\}}{\sqrt{2}}$ & 0 \\
\hline Code 5 & $\frac{s_{1} \exp \left\{j \theta_{1}\right\}}{\sqrt{2}}$ & 0 & $\frac{s_{1} \exp \left\{j \theta_{2}\right\}}{\sqrt{2}}$ \\
\hline Code 6 & $\frac{s_{1} \exp \left\{j \theta_{2}\right\}}{\sqrt{2}}$ & 0 & $\frac{s_{1} \exp \left\{j \theta_{1}\right\}}{\sqrt{2}}$ \\
\hline Code 7 & $\frac{s_{1} \exp \left\{j \theta_{2}\right\}}{\sqrt{2}}$ & $\frac{s_{1} \exp \left\{j \theta_{1}\right\}}{\sqrt{2}}$ & 0 \\
\hline Code 8 & 0 & $\frac{s_{1} \exp \left\{j \theta_{2}\right\}}{\sqrt{2}}$ & $\frac{s_{1} \exp \left\{j \theta_{1}\right\}}{\sqrt{2}}$ \\
\hline
\end{tabular}

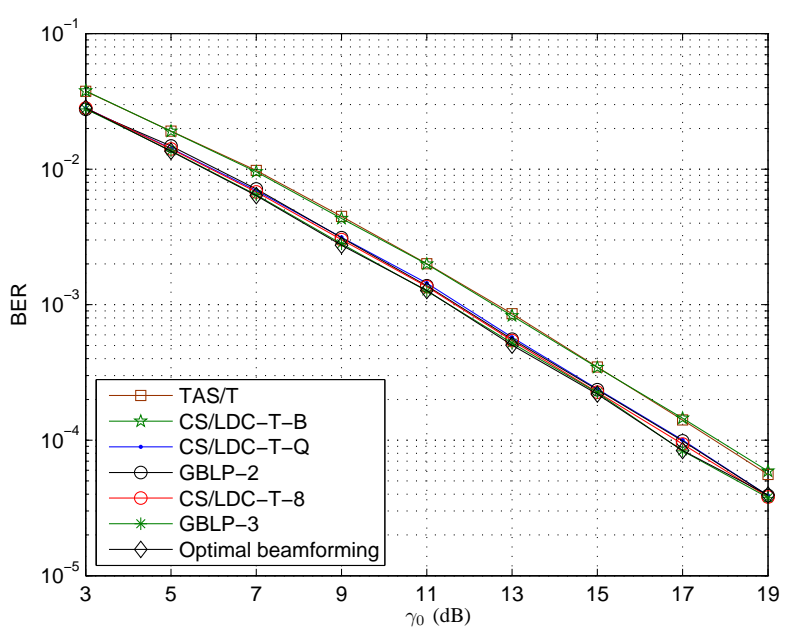

Fig. 1. BER of TAS/T for $b_{f}=1, \mathrm{CS} / \mathrm{LDC}-\mathrm{T}$, for $b_{f}=1,2,3$, GBLP2, GBLP-3, for $b_{f}=2,3$, and the optimal beamforming curve, $M_{T}=2$, $M_{R}=1$

where $\alpha_{1}$ belongs to $2^{b_{f}}$-PSK. The maximum diversity order (equal to 2) is achieved for any number of feedback bits.

In Figure 2, TAS/A [9], TAS/T [10], the GBLP-4 [6], and the new CS/LDC-T-BB, for $b_{f}=2$ feedback bits; the GBLP5 [6] and the new CS/LDC-T-BQ, for $b_{f}=3$ feedback bits; the HS 3Tx 4Fb [12] and the new CS/LDC-T-QQ, for $b_{f}=4$ feedback bits, are considered.

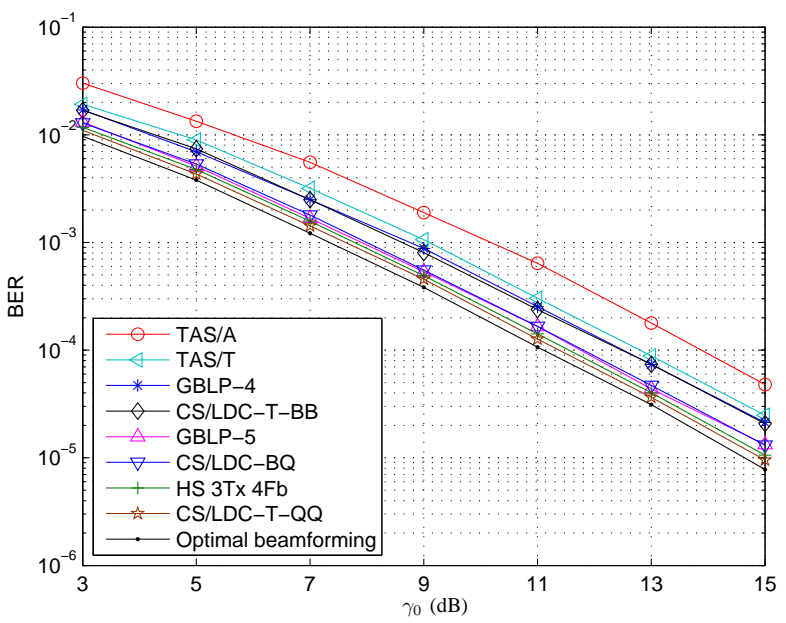

Fig. 2. BER of TAS/A, TAS/T, GBLP-4, and CS/LDC-T-BB for $b_{f}=2$, GBLP-5 and CS/LDC-T-BQ for $b_{f}=3$, HS 3Tx 4Fb and CS/LDC-T-QQ for $b_{f}=4$, and the optimal beamforming curve, $M_{T}=3, M_{R}=1$.
The CS/LDC-T-BB scheme is described by (22) for $b_{f}=2$. In other words, for this scheme the set of LDCs is defined as the four matrices of the form $\left[\begin{array}{lll}s & \alpha_{1} s & \alpha_{2} s\end{array}\right]$, where $\alpha_{1}$ and $\alpha_{2}$ belong to $\{ \pm 1\}$ (BPSK). The CS/LDC-T-BQ scheme, not described before, corresponds to the selection of the best code from the set of LDCs $\left[\begin{array}{lll}s & \alpha_{1} s & \alpha_{2} s\end{array}\right]$, where $\alpha_{1}$ belongs to $\{ \pm 1\}$ (BPSK) and $\alpha_{2}$ belongs to $\{ \pm 1, \pm j\}$ (QPSK). The CS/LDCT-QQ scheme is described by (22) and corresponds to the

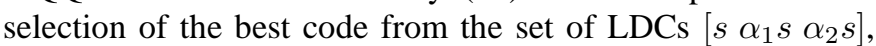
where $\alpha_{1}$ and $\alpha_{2}$ belong to $\{ \pm 1, \pm j\}$ (QPSK). The maximum diversity order (equal to 3 ) is achieved by all the schemes.

From the simulation results presented in this section, it may be observed that the proposed LDCs present the best error performance. It is important to mention that, in some cases, the BER performance of the proposed LDCs are equivalent to the other schemes already presented in the literature, e.g., in [6].

\section{Results: Non-Ideal FeEdback Channel}

The results in Section VI address the limitation to the amount of information that may be sent through the feedback channel, but they consider that an instant error-free feedback channel is available, which clearly does not correspond to reality. LDCs are usually employed in multicarrier systems, and, ideally, the optimum transmission index would be available for each resource element i.e. for each subcarrier and OFDM symbol. In real systems, however, the feedback channel is not perfect and has a limited bandwidth, and the available index may be impaired by several reasons, such as the feedback delay, the transmission of the indexes in regular time periods and the transmission of one single index for a group of subcarriers. Moreover, there is always the possibility that errors may occur in the transmission of the feedback symbols.

In order to study each of these limitations, the LDC set presented in (18) was chosen, with dimensions $M_{T}=2 \times \tau=1$, $Q=1$ symbol per block, and $b_{f}=1$ feedback bit. An OFDM system with $N_{c}=1024$ subcarriers, with subcarrier spacing $\Delta_{f}=10.937 \mathrm{kHz}$, operating at $2.5 \mathrm{GHz}$ was considered. The transmission performance was simulated over a multipath channel following ITU-Pedestrian-B model [26] with $3 \mathrm{~km} / \mathrm{h}$ velocity. The channel responses at the $M_{T}=2$ transmit antennas are uncorrelated and the system uses only $M_{R}=1$ receive antenna. Transmission is done in blocks of $N_{s}=50$ OFDM symbols, which are transmitted in regular intervals of $T=5 \mathrm{~ms}$, which is, for instance, the frame length of a WiMAX system.

In duplex systems one must take into account that part of the available bandwidth in a given transmission direction must be reserved for a feedback channel, which transmits channel state information or the LDC index to be used in the opposite link. This signalling overhead affects negatively the effective achievable data goodput. In order to assess the impact of this overhead, we consider a duplex system with identical transmission schemes, where part of the data in each direction contains feedback information for the opposite direction. Then, it is possible to define the achievable goodput $G$ as

$$
G=\frac{\left(N_{s} N_{c} \log _{2} M-b_{f} / N_{p}\right) C\left(p_{b}\right)}{T} \text { bits/s, }
$$


where $M$ denotes the modulation order, as in M-PSK or MQAM. The term $N_{s} N_{c} \log _{2} M$ indicates the number of bits in one transmission block of $N_{s}$ symbols and $N_{c}$ subcarriers. Of these bits, $b_{f}$ bits are used every $N_{p}$ frames to indicate the best code for the opposite link. Data bits are transmitted with an uncoded bit error probability $p_{b}$, and error-free transmission can ideally be obtained by means of coding with a rate $C\left(p_{b}\right)$, which is the capacity of a binary symmetric channel:

$$
C\left(p_{b}\right)=\left(1-p_{b}\right) \log _{2}\left(2\left(1-p_{b}\right)\right)+p_{b} \log _{2}\left(2 p_{b}\right) .
$$

Computer simulations were used to evaluate the BER and the goodput $G$ in high SNR conditions, where LDC presents higher gains. A signal-to-noise ratio of $20 \mathrm{~dB}$ was used in every simulation, and the results are presented in the following subsections for different feedback channel impairments. Comparisons are made with systems without transmit diversity and with the Alamouti scheme, which do not require any feedback channel. This comparison is relevant for us to investigate if the better performance of LDCs in terms of BER compensates the goodput loss caused by the increased signalling load with LDC. We do not compare the other LDC proposals presented in Section VI, as they present similar or worse performance in terms of BER.

\section{A. Periodic Transmission of the Indices}

We first consider a system in which the feedback information is transmitted at intervals of $N_{p}$ frames, what is usually done in real systems to reduce the signalling load. The receiver selects the best LDC index $i^{*}$ for each OFDM subcarrier according to the criterion in (13), taking into account the instantaneous channel condition at the end of the latest received frame. This means that while the transmitter only updates the index $i^{*}$ at every $N_{p} \times T$ seconds, the channel, and, consequently, the optimal index, may change faster during this interval.

As expected, and may be observed in Figure 3, the BER increases with increasing values of $N_{p}$. It may also be observed that the BER value stabilizes as $N_{p}$ tends to infinity. This happens because the information sent by the feedback channel becomes inaccurate if the LDC index is not updated frequently enough, to follow the channel temporal variations. Considering that for a Doppler frequency of approximately 7 $\mathrm{Hz}$, the coherence time is about $140 \mathrm{~ms}$, one notice that $N_{p}$ approaches 20 frames, the correlation between the channel state immediately before the next data transmission and the channel state at the moment that the receiver chose the index is very low, yielding results that approach those obtained with a random choice of indices.

We can also verify that, when BPSK is used, if $N_{p}>5$ the error rate is worse than the error rate with Alamouti technique [27]. When 8PSK is used, the same happens when $N_{p}>15$. Again, it is interesting to remember that the Alamouti scheme is one particular case of LDC with no need for feedback, and it performs better than a random LDC. Nevertheless, we can observe that the BER with LDC is always lower than for a system without diversity, even for very long feedback periods, i.e., LDCs still provide some diversity, even with a non-optimal index.

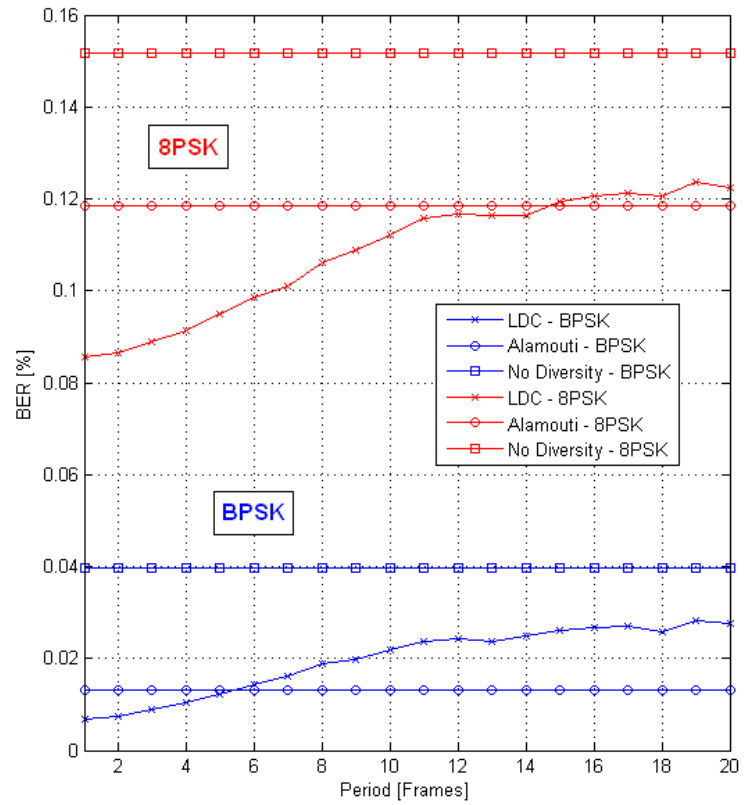

Fig. 3. BER with varying signalling period, at $\mathrm{SNR}=20 \mathrm{~dB}$.

Figure 4 shows that the goodput $G$ presents a peak value at $N_{p}=2$ for BPSK. This happens because, while, the error rate increases with larger periods, the feedback overhead, on the other hand is reduced, resulting in higher usable transmission rate. So, it can be observed that, although the LDC has a lower BER than Alamouti when $N_{p}<5$, the goodput performance of LDC is only slightly better than the one of Alamouti scheme when BPSK in used for $N_{p}<4$. Also, when evaluating the goodput with 8PSK, it is verified that the LDC scheme performs up to $20 \%$ better than Alamouti if $N_{p}<15$, and short periods should be employed. Thus, higher gains may be achieved when the modulation order is increased, as the overhead becomes proportionally lower.

\section{B. Feedback Channel Delay}

Besides the periodical feedback, one must also account for the delay in this information. The receiver must estimate the channel, process it, choose the best index, and wait for the next transmission opportunity. To investigate the impact of this delay, we now consider a feedback period $N_{p}=1$, but with a delay of $N_{d}$ frames. The receiver selects and sends the LDC index $i^{*}$ for each frame and subcarrier, but the transmitter only receives the data $T N_{d}$ seconds later.

In Figure 5 it is possible to see that the BER increases with larger $N_{d}$ in both BPSK and 8PSK. Using BPSK, the error rate with LDC is better than Alamouti's if $N_{d}<2$. If 8PSK is used, the error rate is better for $N_{d}<5$.

Again, it is possible to observe that the BER curve tends to a constant value when $N_{d}$ increases, for the same reason as in the previous subsection.

In terms of the goodput $G$, in Figure 6, we notice that, for BPSK modulation, the LDC technique is worse than Alamouti's for any positive feedback channel delay. For 8PSK modulation, it is interesting to use the LDC technique if $N_{d}<5$. 


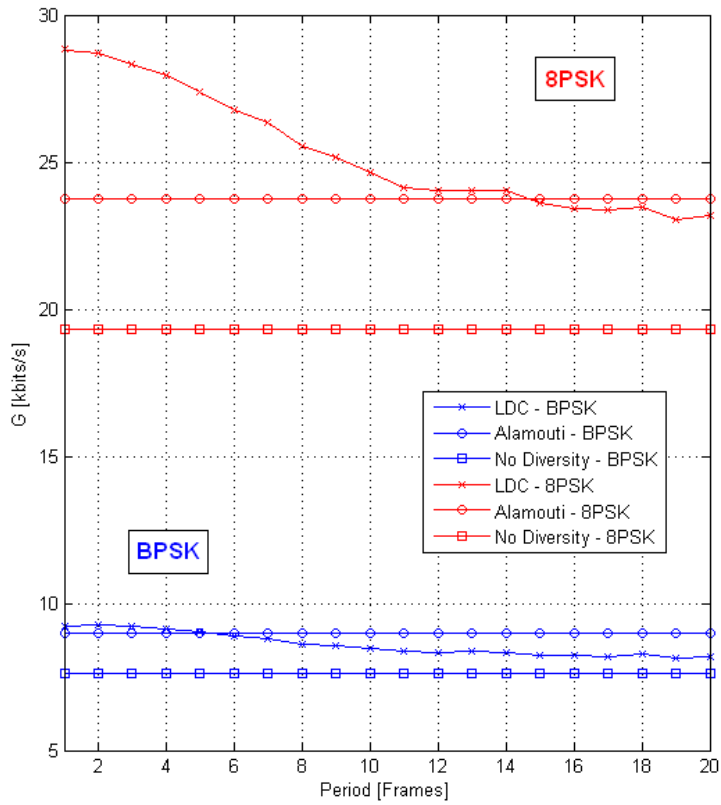

Fig. 4. Goodput with varying signalling period.

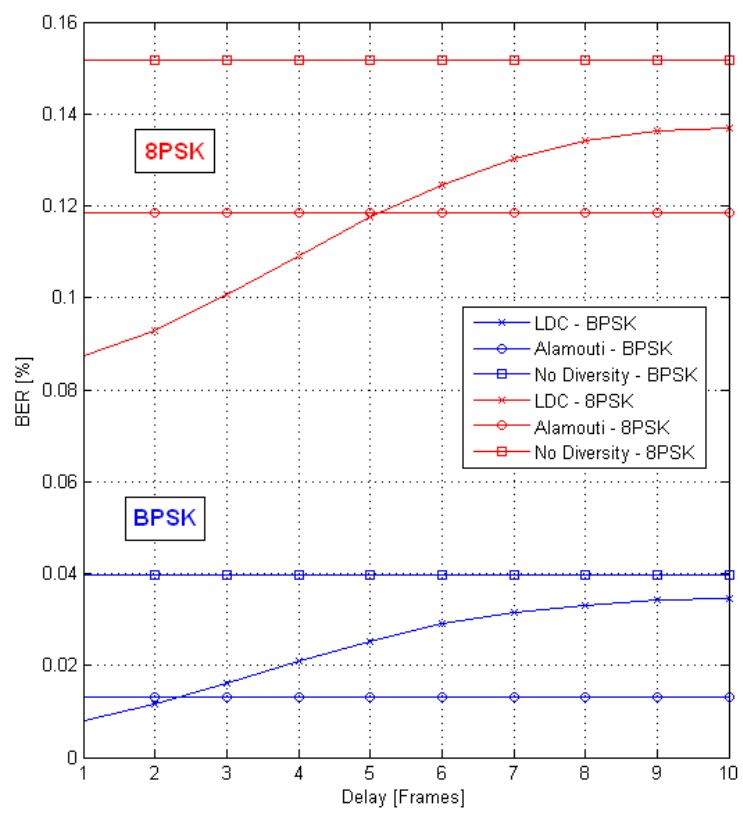

Fig. 5. BER with varying feedback delay.

\section{Subcarrier Grouping}

In OFDM systems it is safe to assume that neighboring subcarriers have highly correlated channel responses, and, thus, the feedback information can be sent for groups of $N_{c}$ adjacent subcarriers in order to reduce the signalling load. This is investigated in this subsection. We consider here $N_{d}=0$ and $N_{p}=1$. The same index $i^{*}$ is used by the transmitter for all subcarriers within a group, and this index is chosen based on the channel of the central subcarrier. Different groups may be assigned different indices.

It is possible to observe in Figure 7 that, as expected, BER grows with the increase of the number of subcarriers per group.

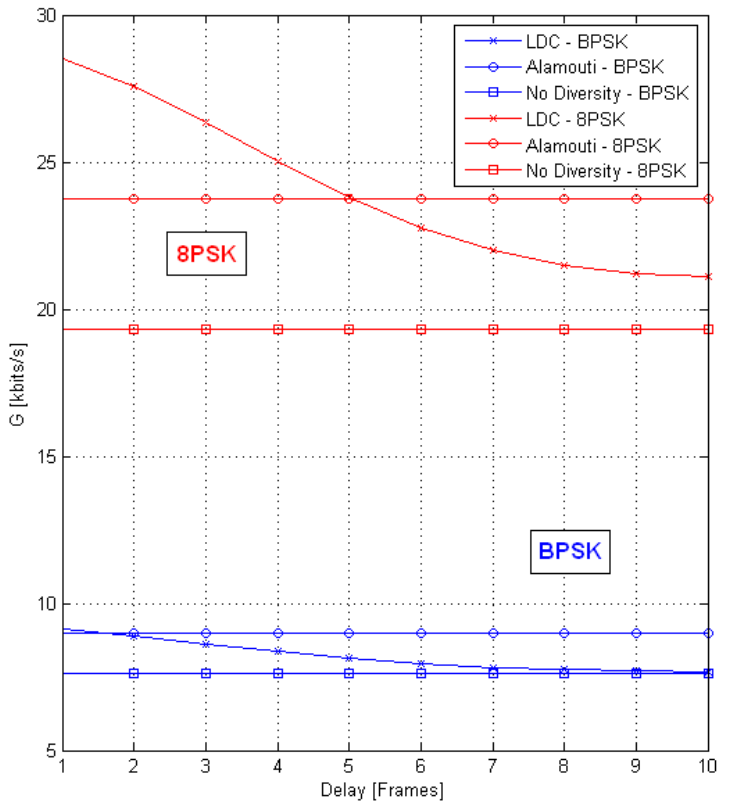

Fig. 6. Goodput with varying feedback delay.

However, this increase is only considerable when $N_{c}>8$. When goodput $G$ is verified, in Figure 8, it is possible to note that the goodput remains stable until $N_{c}=16$. Therefore, an ideal number of subcarriers per group may be chosen depending on channel characteristics. However, the figures show that the subcarrier grouping is not the most impacting limitation, and relatively large groups can be used.

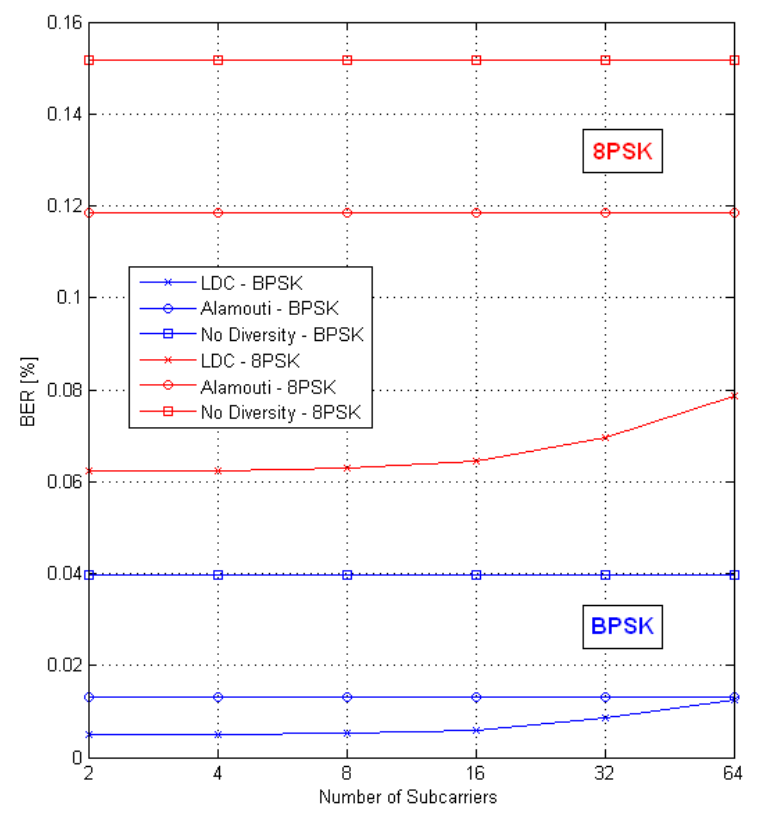

Fig. 7. BER with varying subcarrier group sizes.

\section{Feedback channel errors}

We consider now a system with $N_{d}=0, N_{p}=1$ and $N_{c}=1$, but with a probability $P_{f}$ that an error occur in 


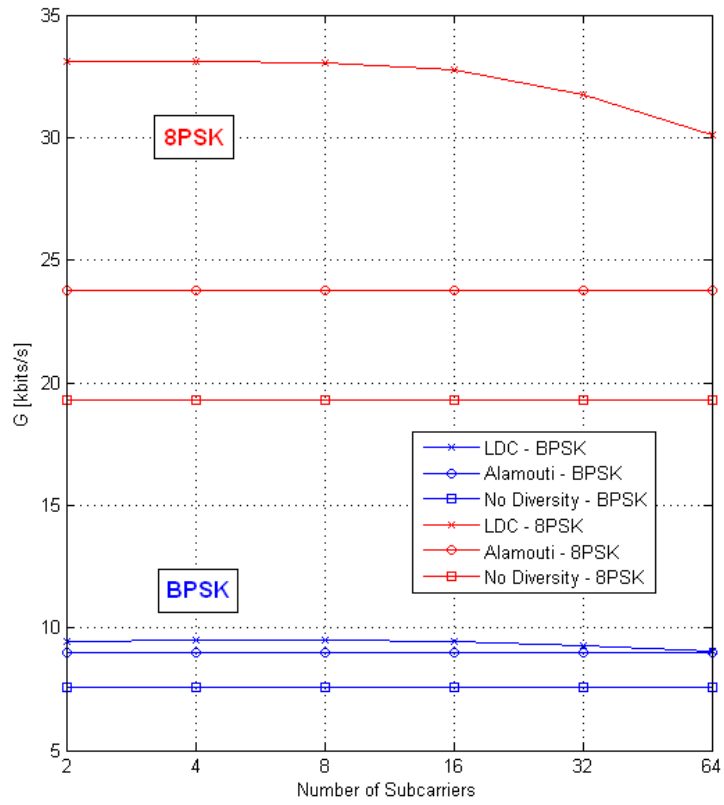

Fig. 8. Goodput with varying subcarrier group sizes.

the transmission over the feedback channel. Thus, there is a possibility that the transmitter uses wrong data to select the LDC that will be used to send the next block. In Figure 9 it is possible to notice that the gain of the LDC technique is reduced when $P_{f}$ increases. Also, when BPSK is in use, in case $P_{f}>0.2$, it is better to use Alamouti than LDC technique with feedback. In case 8PSK is used, this will happen only when $P_{f}>0.5$. Clearly, when $b_{f}=1$, as in this case study, the error probability is likely to be limited to $P_{f}=0.5$.

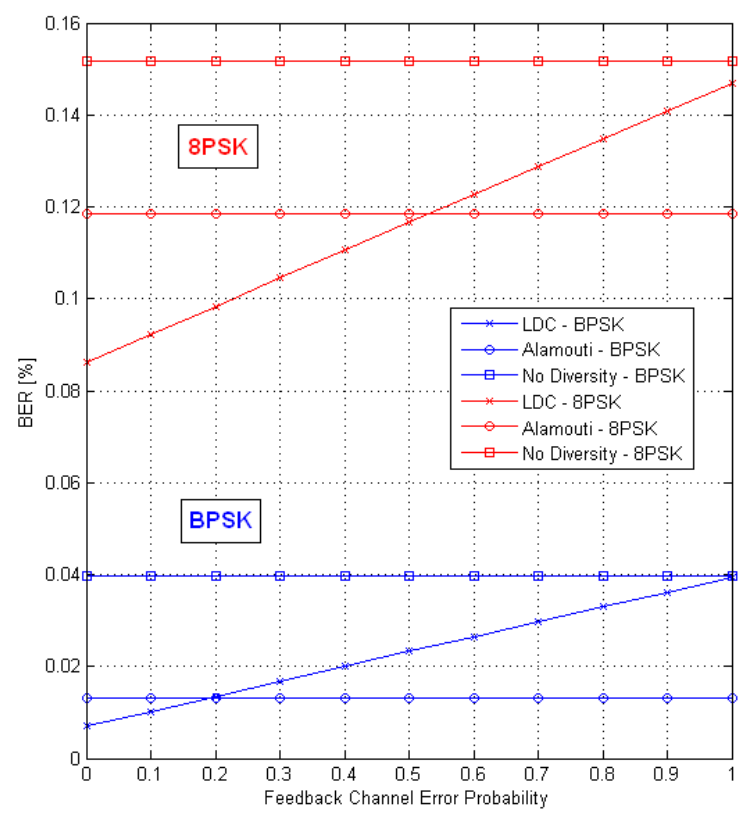

Fig. 9. BER with varying feedback error probability.

When the goodput $G$ is verified in Figure 10, it is observed that, when BPSK is used, the use of the LDC technique is only recommended for error rates below 0.1 , but even then with very small gains. When 8PSK modulation is used, this technique is considerably better than Alamouti when $P_{f}$ is less than about 0.5 .

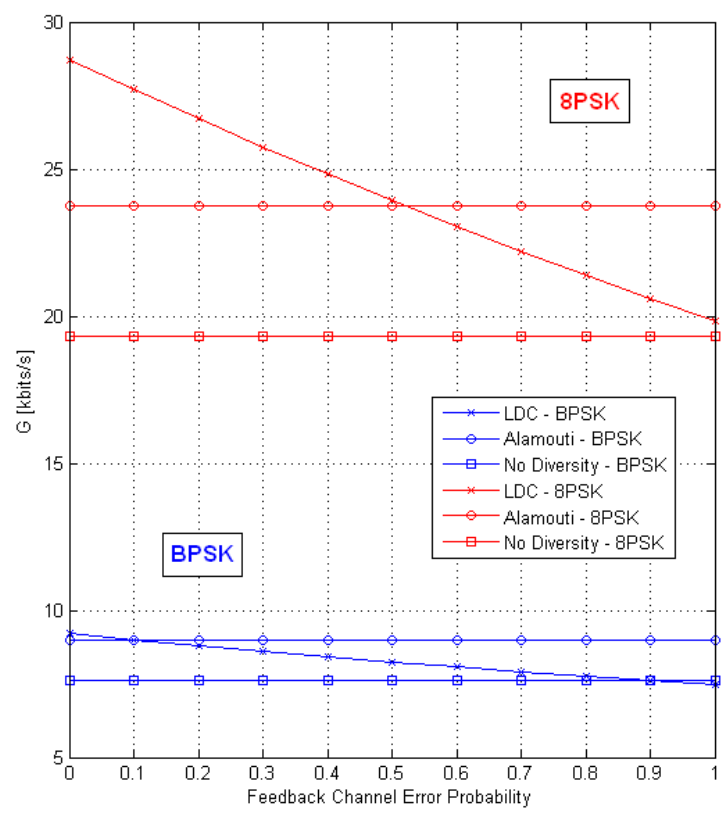

Fig. 10. Goodput with varying feedback error probability.

It is important to highlight, however, that, in all studied cases, no channel coding was considered in the feedback channel. In practical systems, the feedback information is channel coded to avoid signalling errors. This is achieved at the expense of an increased overhead. In any case, since in our application only a few bits are sent over the feedback channel, the cost of such overhead can be considered as negligible.

One should bear in mind that the results presented in this section should not be viewed quantitatively, but rather as performance trends when feedback impairments are considered. In a real-life system, the optimum feedback delay, the length of the feedback period, and the size of each subcarrier group depend on the channel coherence band and interval, as well as on the employed modulation and coding scheme and target error rate. However, in any situation it is necessary to weigh the pros and cons of using LDCs, considering not only their performances under ideal conditions, but also the signalling overhead and robustness in the presence of nonideal feedback, and, in some situations, schemes that do not require feedback, such as Alamouti's, may be more efficient. The increased complexity of LDCs must also be taken into account, as the choice of the optimum index, as stated in (13), may be computationally demanding.

\section{CONCLUSIONS}

In this paper, we proposed a general framework for transmit code selection with linear dispersion codes. Based on the instantaneous channel conditions, the transmitter chooses an LDC from a set of LDCs aiming to minimize the error probability. The determination of the best set of LDCs, i.e., the one that minimizes the average error probability, was described as a constrained optimization problem. For certain 
parameters we presented good sets of LDCs, obtained from an optimization algorithm. We note that all sets of LDCs presented in Section $\mathrm{V}$ are surprisingly simple, rendering the feedback bits to carry phase information only. This is always going to be the case when the parameters match, e.g., $\tau=Q$ and $b_{f}=\left(M_{T}-1\right) b$. Other extensions are possible. For example, when $\tau=Q$ and $b_{f}=\sum_{i=1}^{M_{T}-1} b_{i}$, where $b_{i}$ is a positive integer, the signals transmitted through antenna $i+1$ are rotated by a phase in $2^{b_{i}}$-PSK, depending on the value of the feedback bits. This represents a more general solution as compared to previously published Q-EGT. In all of these cases, the best sets of LDCs following the idea in Section V are likely to be the optimal sets. However, for $b_{f}<M_{T}-1$, the optimal solution does not seem to be so straightforward, and may involve the transmission of mixed symbols through some of the antennas. After the study of a set of LDCs in such conditions that the feedback channel is submitted to the limitations that usually challenge real systems, it was observed that the feasibility of these systems depends on signalling characteristics, such as the period, the delay and the number of subcarriers per group, as well as the used modulation, and these limitations depend on the channel characteristics, such as coherence time and band. Furthermore, we also showed that not only the error rate, but also the goodput must be considered since, in practice, it will be reduced by the frequent signalling. Feedback schemes with LDCs are a promising technique to improve performance in multiple antenna systems, but their use must be analyzed carefully for each deployment scenario.

Future work in this topic includes the search for a less complex algorithm, which is urgently needed for systems with more antennas and more feedback bits, or for spacetime-frequency codes, in which case the proposed algorithm becomes quickly unfeasible. It is also not clear if these codes are still optimal for other channels, such as those with antenna correlation or a line-of-sight component, particularly when some of the design restrictions are removed. Finally, a study to determine the ideal set size and element values regarding the search space discussed in Section IV should also be considered as future work.

\section{ACKNOWLEDGEMENTS}

This work was supported in part by the Brazilian Agencies FAPERGS and CNPq.

\section{REFERENCES}

[1] V. Tarokh, N. Seshadri, and A. R. Calderbank, "Space-time codes for high data rate wireless communications: Performance criterion and code construction," IEEE Trans. Inf. Theory, vol. 44, pp. 744-765, March 1998.

[2] T. K. Y. Lo, "Maximum ratio transmission," IEEE Trans. Commun., vol. 47, no. 10, pp. 1458-1461, October 1999.

[3] D. J. Love and R. W. Heath Jr., "Equal gain transmission in multipleinput multiple-output wireless systems," IEEE Trans. Commun., vol. 51, no. 7, pp. 1102-1110, July 2003 .

[4] A. Narula, M. J. Lopez, M. D. Trott, and G. W. Wornell, "Efficient use of side information in multiple-antenna data transmission over fading channels," IEEE J. Sel. Areas Commun., vol. 16, no. 8, pp. 1423-1436, October 1998

[5] R. W. Heath Jr. and A. Paulraj, "A simple scheme for transmit diversity using partial channel feedback," in Proc. 32nd Asilomar Conf. on Signals, Systems, and Computers, Pacific Grove, California, November 1998, pp. 2441-2444
[6] D. J. Love and R. W. Heath Jr., "Grassmannian beamforming for multiple-input multiple-output wireless systems," IEEE Trans. Inf. Theory, vol. 49, no. 10, pp. 2735-2747, October 2003.

[7] C. R. Murthy and D. Rao, "Quantization methods for equal gain transmission with finite rate feedback," IEEE Trans. Signal Processing, vol. 55, no. 1, pp. 233-245, January 2007.

[8] K. K. Mukkavilli, A. Sabharwal, E. Erkip, and B. Aazhang, "On beamforming with finite rate feedback in multiple-antenna systems," IEEE Trans. Inf. Theory, vol. 49, no. 10, pp. 2562-2579, October 2003.

[9] D. Gore and A. Paulraj, "MIMO antenna subset selection with spacetime coding," IEEE Trans. Signal Processing, vol. 50, no. 10, pp. 2580 2588, December 2002.

[10] Z. Chen, J. Yuan, and B. Vucetic, "Analysis of transmit antenna selection/maximal-ratio combining in Rayleigh fading channels," IEEE Trans. Vehic. Tecnology, vol. 54, no. 4, pp. 1312-1321, July 2005.

[11] R. Machado and B. F. Uchôa-Filho, "Space-time block coding with hybrid transmit antenna/code selection," in Proc. IEEE Int. Conf. on Commun. (ICC), Paris, France, June 2004, pp. 819-822.

[12] S. Şahin, M. E. Çelebi, and . Aygölü, "Space diversity schemes with feedback for three transmit antennas," AEÜ-International Journal of Electronics and Communications, vol. 60, no. 8, pp. 613-617, September 2006.

[13] R. Machado and B. F. Uchôa-Filho, "Extended techniques for transmit antenna selection with STBCs," IEEE Journal of Communication and Information Systems (JCIS), vol. 21, no. 3, pp. 188-195, December 2006.

[14] J. Akhtar and D. Gesbert, "Partial feedback based orthogonal block coding," in Proc. IEEE Vehicular Technology Conf. (VTC-Spring), Orlando, Florida, April 2003, pp. 287-291.

[15] — - "Extending orthogonal block codes with partial feedback," IEEE Trans. Wireless Commun., vol. 3, no. 6, pp. 1959-1962, November 2004.

[16] R. Machado, R. dos Santos e B. F. Uchôa-Filho, "Sobre os códigos grupo-coerentes," in Proc. XXII Simpósio Brasileiro de Telecomunicações (SBrT'05), Campinas, Brasil, Setembro 2005.

[17] V. Tarokh, H. Jafarkhani, and A. Calderbank, "Space-time block codes from orthogonal designs," IEEE Trans. Inform. Theory, vol. 45, no. 5, pp. 1456-1467, July 1999.

[18] B. Hassibi and B. Hochwald, "High-rate codes that are linear in space and time," IEEE Trans. Inf. Theory, vol. 48, no. 7, pp. 1804-1824, July 2002.

[19] R. Machado, B. F. Uchôa-Filho, and T. M. Duman, "Linear Dispersion Codes for MIMO Channels with Limited Feedback," Proc. IEEE Wireless Commun. and Networking Conf. (WCNC), pp. 199-204, April 2008.

[20] G. J. Foschini, "Layered space-time architecture for wireless communication in a fading environment when using multiple antennas," Bell Labs. Tech, Journal, vol. 1, no. 2, pp. 41-59, Autumn 1996.

[21] M. O. Damen, A. Chkeif, and J. C. Belfiore, "Lattice code decoder for space-time codes," IEEE Commun. Lett., vol. 4, no. 5, pp. 166-169, May 2000.

[22] B. Dong, X. Wang, and A. Doucet, "A new class of soft MIMO demodulation algorithms," IEEE Trans. Signal Process., vol. 51, no. 11, pp. 2752-2763, November 2003.

[23] G. J. Foschini, G. Golden, R. Valenzuela, and O. Wolniansky, "Simplified processing for high spectral efficiency wireless communication employing multi-element arrays," IEEE J. Sel. Areas Commun., vol. 17, no. 11, pp. 1841-1852, November 1999.

[24] D. Gesbert, "Minimum-error linear receivers for ill-conditioned MIMO channels," in Proc. IEEE Workshop Signal Process. Advances in Wireless Commun. (SPAWC), Rome, Italy, June 2003, pp. 462-466.

[25] X. Wang, V. Krishnamurthy, and J. Wang, "Stochastic gradient algorithms for design of minimum error-rate linear dispersion codes in MIMO wireless systems," IEEE Trans. Signal Process, vol. 54, no. 4, pp. 1242-1255, April 2006.

[26] ITU ITU-R M.1225, "Guidelines for evaluations of radio transmission technologies for imt-2000," 1997.

[27] S. M. Alamouti, "A simple transmit diversity technique for wireless communications," IEEE J. Select. Areas Commun, vol. 16, pp. 14511458, October 1998. 


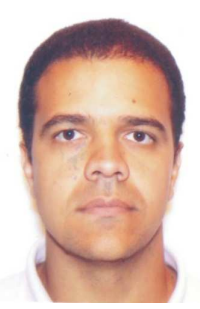

Advanced.
Fernando Menezes Leitão Tavares received his electrical engineer degree and his M.Sc. degree from the Universidade de Brasília (UnB) in 2005 and 2009, respectively. He also worked at Nokia Institute of Technology (INdT), Brasília, DF, Brazil as a researcher for nearly four years. He is currently working toward a Ph.D. degree at Aalborg University, Denmark, in close cooperation with Nokia Siemens Networks. His current research interests include radio resource management, software defined radio and concept development for 3GPP LTE-

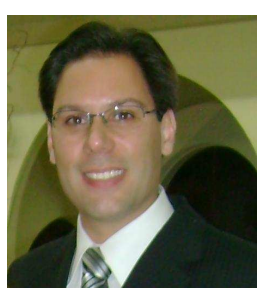

Renato Machado ( $\left.\mathrm{S}^{\prime} 04-\mathrm{M}^{\prime} 08\right)$ was born in Jaú, SP, Brazil, in 1979. He received the B.S.E.E. degree from the São Paulo State University (UNESP), Ilha Solteira, SP, Brazil, in 2001. He received the M.Sc. degree and the Ph.D. degree in electrical engineering from the Federal University of Santa Catarina (UFSC), Florianópolis, SC, Brazil, in 2004 and 2008, respectively. He was a Visiting Researcher in the Department of Electrical Engineering, Arizona State University (ASU), Tempe, AZ, U.S.A., from August 2006 to June 2007. He was a Research Engineer in the Nokia Institute of Technology, Brazil, from October 2007 to March 2008. He was a Visiting Professor in the Department of Electrical Engineering, Federal University of Juiz de Fora, MG, Brazil, from September 2008 to August 2009. Since August 2009 he has been an Assistant Professor in the Federal University of Santa Maria, RS, Brazil. His research interests include MIMO systems, space-time coding, linear dispersion codes, cooperative communication, wireless communications, and power line communications.

Dr. Machado is a member of the IEEE Communications Society, and the Brazilian Telecommunications Society.

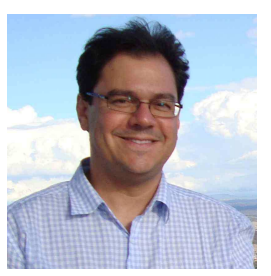

Bartolomeu F. Uchôa-Filho (S'94-M'96) was born in Recife, Brazil, in 1965. He received the B.S.E.E. degree from the Federal University of Pernambuco (UFPE), Recife, Brazil, in 1989; the M.S.E.E. degree from the State University of Campinas (UNICAMP), Campinas, Brazil, in 1992; and the Ph.D. degree in electrical engineering from the University of Notre Dame, Notre Dame, Indiana, U.S.A., in 1996. During 1997-1999 he held a Post-Doctoral position at the State University of Campinas. From August 1999 to January 2000 he was a Visiting Researcher in the Department of Electrical Engineering, Federal University of Santa Catarina, Florianópolis, Brazil. Since February 2000 he has been an Associate Professor in the same Department. From March 2009 to February 2010, he was a PostDoctoral Fellow at the University of Sydney, Australia. His research interests are in the area of coding and information theory, with applications to digital communications systems.

Dr. Uchôa-Filho is a member of Eta Kappa Nu, the IEEE Information Theory Society, the IEEE Communications Society, ARC Communications Research Network (ACoRN, Austrália), and the Brazilian Telecommunications Society. For the 2003-2004 period, he served as the Editor-in-Chief of the Journal of the Brazilian Telecommunications Society. He was the Technical Program Committee Chair of the 27th Brazilian Telecommunications Symposium (SBrT'09). 